\title{
Durability of switch regimens based on rilpivirine or on integrase inhibitors, both in association with tenofovir and emtricitabine, in HIV-infected, virologically suppressed patients
}

Nicola Gianotti ${ }^{1 *}$ (D), Andrea Poli ${ }^{1}$, Silvia Nozza ${ }^{1}$, Laura Galli ${ }^{1}$, Nadia Galizzi ${ }^{1,2}$, Marco Ripa ${ }^{1,2}$, Marco Merli ${ }^{1}$, Alessia Carbone ${ }^{1}$, Vincenzo Spagnuolo ${ }^{1,2}$, Adriano Lazzarin ${ }^{1,2}$ and Antonella Castagna ${ }^{1,2}$

\begin{abstract}
Background: Switch strategies based on rilpivirine/tenofovir/emtricitabine or on an integrase inhibitor (InSTI) plus tenofovir/emtricitabine have never been compared in randomized clinical trials. The main aim of the study was to investigate the durability of these two switch regimens in virologically suppressed, HIV-infected patients.

Methods: Retrospective analysis of patients who started rilpivirine or an InSTI (both with tenofovir and emtricitabine) with $<50$ HIV-RNA copies/mL and had at least one HIV-RNA assessed while receiving the study regimen. Virological failure (VF) was defined as two consecutive measurements of HIV-RNA >50 copies/mL. Treatment failure (TF) was define as either VF or discontinuation of any drug of the regimen. Durability was assessed by the Kaplan-Meier method and compared by Log-rank test. Residual viremia was defined as any detectable HIV-RNA below 50 copies $/ \mathrm{mL}$, as assed by a real-time PCR assay.
\end{abstract}

Results: Six hundred seventy-five patients (466 switched to a rilpivirine-, 209 switched to an InSTI-based regimen [18\% dolutegravir, 39\% raltegravir, 43\% elvitegravir/cobicistat] were included in the analysis.

The median (interquartile range, IQR) follow-up in the rilpivirine and in the InSTI group was 16.7 (8.8-22.2) and 10.4 (5.4-19.6) months. The 1-year cumulative probabilities (95\%Cl) of VF and TF were $0.97 \%(0.36 \%-2.62 \%)$ and 9.73\% $(7.21 \%-13.06 \%)$ in the rilpivirine group and $1.83 \%(0.57 \%-5.77 \%)$ and $8.75 \%(5.25 \%-14.4 \%)$ in the InSTI group, with no difference between groups ( $p=0.328$ and 0.209 for VF and TF). The proportion of time spent with residual viremia was comparable in the two groups (9\% [IQR 0.5\%-49\%] and 17\% [IQR 0.5\%-50\%] in the rilpivirine and in the InSTI group, $p=0.087$ ).

By the multivariable Cox regression model, TF was independently associated with being on therapy with a protease inhibitor vs. a non-nucleoside reverse transcriptase inhibitor at switch (AHR $=0.52 ; 95 \% \mathrm{Cl}=0.31-0.90 ; p=0.018$ ), baseline total/HDL-cholesterol ratio (AHR $=1.19$ per 0.5 -units increments; $95 \% \mathrm{Cl}=1.06-1.34 ; p=0.004$ ), baseline estimated glomerular filtration rate (AHR $=0.78$ per 10-units increments; $95 \% \mathrm{Cl}=0.67-0.90 ; p=0.001$ ) and baseline (Continued on next page)

\footnotetext{
* Correspondence: gianotti.nicola@hsr.it

Work presented in part at the 20th international workshop on HIV

observational databases, 7th - 9th April 2016, Budapest, Hungary, abstract

number 109 and at the VIII Italian conference on AIDS and antiviral research,

Milano, Italy, 6th - 8th June 2016, abstract number OC 75.

'Infectious Diseases, San Raffaele Scientific Institute, Via Stamira d'Ancona 20,

20127 Milan, Italy

Full list of author information is available at the end of the article
} 


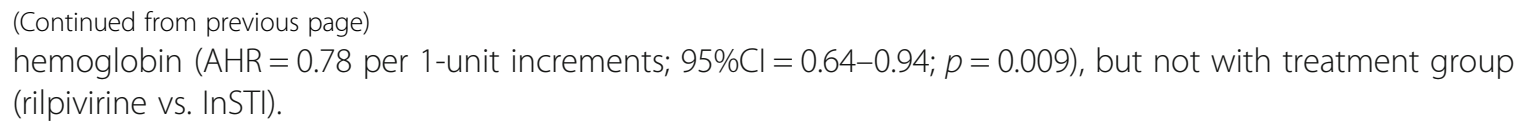

Keywords: Rilpivirine, Raltegravir, Elvitegravir/cobicistat, Dolutegravir, Non-nucleoside reverse transcriptase inhibitors, Integrase inhibitors, Switch regimen, Residual viremia, Virological suppression,

\section{Background}

Randomized clinical trials support the switch both to the fixed dose combination (FDC) of rilpivirine/tenofovir disoproxil fumarate/emtricitabine from a ritonavirboosted protease inhibitor (PI/r) and to regimens based on integrase strand transfer inhibitors (InSTI) from any kind of antiretroviral therapy. In the SPIRIT Study switching from a regimen based on a PI/r to the FDC of rilpivirine/tenofovir/emtricitabine was not inferior to continuing the $\mathrm{PI} / \mathrm{r}$, with virological success in $93,7 \%$ and $89,9 \%$ of patients at 48 weeks [1]. Non-inferiority was shown also for switching from the FDC of efavirenz/tenofovir disoproxil fumarate/emtricitabine to rilpivirine/tenofovir alafenamide/emtricitabine [2], as well as for switching from a PI/ $r$ to raltegravir [3], from a regimen based on a non-nucleoside reverse transcriptase inhibitor (NNRTI) to the FDC of elvitegravir/cobicistat/ emtricitabine/tenofovir disoproxil fumarate [4] or from any regimen to the FDC of dolutegravir/abacavir/lamivudine [5]. By contrast, in the STRATEGY-PI Study, switching from a PI/r to the FDC of elvitegravir/cobicistat/emtricitabine/tenofovir disoproxil fumarate was superior in terms of virological success $(93,8 \%$ vs. $87,1 \%)$ and improved patients related outcomes [6], while switching from lopinavir/ritonavir to raltegravir (without changing the nucleoside backbone) was associated with a higher incidence of virological failure [7].

However, these switch strategies have never been compared in randomized clinical trials and it is very unlikely that such a trial will be performed.

The aim of this study was two-fold: first, we aimed at identifying factors associated with the choice of a FDC of rilpivirine/tenofovir/emtricitabine vs. an InSTI-based switch regimen; secondly, we aimed at investigating the durability of rilpivirine/tenofovir/emtricitabine and of InSTI-based switch regimens (when associated to tenofovir disoproxil fumarate/emtricitabine, TDF/FTC) in virologically suppressed HIV-infected patients in clinical practice.

\section{Methods}

Retrospective cohort study on patients followed at the Infectious Diseases Department of the San Raffaele Scientific Hospital in Milan (Italy). Data recorded in the database of the Infectious Diseases Department of the
San Raffaele Hospital (IDD-HSR) were used for the analyses. At their first visit in our clinic, subjects provide written informed consent to include their clinical and laboratory data in the IDD-HSR for scientific purposes. The study was approved by the ethics committee of the San Raffaele Scientific Institute.

Eligible patients were those who started rilpivirine or an InSTI (both along with TDF and FTC) with $<50$ HIVRNA copies/mL and had at least one HIV-RNA assessed while receiving the study regimen. Patients who switched to one of the study regimens since 2008 (date of availability of raltegravir in Italy) were included in the analyses. Patients were followed up to virological failure (VF) or discontinuation of any drug or data freezing (8th September 2015), whichever occurred first.

Estimated glomerular filtration rate (eGFR) was calculated using the Chronic Kidney Disease Epidemiology Collaboration Equation (CKD-EPI) [8] and the liver fibrosis FIB-4 index was calculated as described [9].

HIV-RNA was quantified by using the kinetic PCR molecular system (kPCR, Versant HIV-1 RNA kPCR 1.0; Siemens HealthCare Diagnostics, Tarrytown, NY, USA) up to March 2014 and by using the Abbot Real-Time PCR (Abbott Molecular, Des Plaines, IL, USA) thereafter. The kPCR assay gives three possible outputs: (i) a quantitative result for HIV-RNA values of $\geq 37$ copies $/ \mathrm{mL}$; (ii) a semiquantitative result (detectable below 37 copies $/ \mathrm{mL}$ ) when HIV RNA is detectable but not precisely quantifiable; (iii) a qualitative result ('undetectable') when no signal can be detected. The Abbot Real-Time PCR assay gives also three possible outputs: (i) a quantitative result for HIV RNA values of $\geq 40$ copies $/ \mathrm{mL}$; (ii) a semiquantitative result (detectable below 40 copies $/ \mathrm{mL}$ ) when HIV RNA is detectable but not precisely quantifiable; (iii) a qualitative result ('undetectable') when no signal can be detected.

VF was defined as two consecutive measurements of HIV-RNA $>50$ copies/mL or a single HIV-RNA $>50$ copies/mL followed by ART modification; an unconfirmed HIV-RNA $>50$ copies $/ \mathrm{mL}$ (i.e. one measurement $>50$ copies $/ \mathrm{mL}$ preceded and followed by a measurement $<50$ copies $/ \mathrm{mL}$ ), not followed by ART modification, was defined as viral blip. Residual viremia was defined as any detectable HIV-RNA below 50 copies/mL, as assed by Siemens kPCR or by Abbot Real-Time PCR. 
Time spent with residual viremia was calculated as a proportion of time with residual viremia on observed follow-up. If between 2 observation the viremia changed from undetectable to residual or vice-versa, the time spent considered in this interval was the half. The mathematical formula was:

$$
T_{\%}=\frac{\sum_{i=1}^{i=j}\left(\frac{t_{i}-t_{i-1}}{a}\right)}{t_{t o t}} \cdot 100
$$

where $t$ is the time length of interval $i, j$ is the last observation and $t_{t o t}$ is the patient's cumulative follow-up. If, during the $i^{t h}$ interval, viremia changed from undetectable to residual or vice-versa, then $a=2$, else $a=1$.

Treatment failure (TF) was define as either VF or discontinuation (for any length) of any drug of the regimen, for any reason. Causes of change in the regimen were reviewed independently by two clinicians; discordances were discussed and reconciled.

Descriptive data are expressed as median (interquatile range) of frequency (\%), as appropriate.

Chi-square and Mann-Whitney tests were used to evaluate differences between the two groups for categorical and continuous variables respectively. Durability was assessed by the Kaplan-Meier curve and compared by Log-rank test. Multivariable logistic regression was used to identify predictors of opting for an InSTI- rather than a rilpivirine-based regimen and a multivariable Cox regression model was used to identify factors independently associated with TF.

All of the statistical tests were two-sided at 5\% level, and were performed using SAS Software (release 9.2; SAS Institute).

\section{Results}

Six hundred seventy-five patients (466 switched to a rilpivirine-, 209 switched to an InSTI-based regimen [18\% dolutegravir, 39\% raltegravir, 43\% elvitegravir/cobicistat]), on antiretroviral therapy since 6.6 [3.3-14.1] years and with HIV-RNA <50 copies/mL since 3.1 [1.1-5.6] years, were included in the analysis; their baseline characteristics are illustrated in Table 1 and in the Additional file 1: Table S1. Dolutegravir was used at a daily dose $50 \mathrm{mg}$ in all cases, raltegravir at the standard dose of $400 \mathrm{mg}$ twice daily and elvitegravir/cobicistat was always co-formulated with TDF/FTC).

After adjusting for age, gender, HIV risk factor, higher HIV-RNA value before starting ART, nadir and baseline CD4+ count, baseline triglycerides and cholesterol, history of failure to nucleoside reverse transcriptase inhibitors (NRTIs) or to non-NRTIs (NNRTIs), opting for an InSTI- rather than a rilpivirine-based regimen was more likely in subjects co-infected with $\mathrm{HCV}(\mathrm{OR}=2.16$; $95 \% \mathrm{CI}=1.26-3.71 ; p=0.015)$, with longer exposure to antiretroviral therapy $(\mathrm{OR}=1.05$ per year longer; $95 \% \mathrm{CI}$ $=1.01-1.09 ; p=0.042)$ and shorter time with undetectable viremia $(\mathrm{OR}=0.93$ per year longer; $95 \% \mathrm{CI}=0.86-$ $0.99 ; p=0.041$ ), treated with protease inhibitors (PIs) vs NNRTIs $(\mathrm{OR}=5.14 ; 95 \% \mathrm{CI}=3.09-8.95 ; p=0.003)$, and treated with regimens not based on a $\mathrm{PI} / \mathrm{r}$ and not based on a NNRTI vs those treated with NNRTIs (OR = 5.67; $95 \% \mathrm{CI}=2.22-14.38 ; p=0.032$ ) .

The median (interquartile range, IQR) follow-up in the rilpivirine and in the InSTI group was 16.7 (8.8-22.2) and 10.4 (5.4-19.6) months. Four (0.9\%) and three (1.4\%) patients showed VF at 1 year in the rilpivirine and in the InSTI group; cumulatively, 12 patients (8 [1.7\%] in the rilpivirine and 4 [1.9\%] in the InSTI group) showed VF during follow-up: their characteristics at VF are detailed in Table 2 . TFs at 1 year were 38 (8.2\%) and $14(6.7 \%)$ in the rilpivirine and in the InSTI group. The 1-year cumulative probabilities $(95 \% \mathrm{CI})$ of $\mathrm{VF}$ and $\mathrm{TF}$ were $0.97 \%(0.36 \%-2.62 \%)$ and $9.73 \%(7.21 \%-13.06 \%)$ in the rilpivirine group and $1.83 \%(0.57 \%-5.77 \%)$ and $8.75 \%(5.25 \%-14.4 \%)$ in the InSTI group, with no difference between groups $(p=0.328$ and 0.209 for VF and TF; Fig. 1, panel A and B).

The incidence rate (IR) $(95 \% \mathrm{CI})$ of viral blips was 4.46 (3.07-6.27) and $4.48(2.51-7.40)$ per 1000 person months of follow-up in the rilpivirine and in the InSTI group $(p=0.988)$.

The proportion of time spent with residual viremia was comparable in the two groups ( $9 \%$ [IQR $0.5 \%-49 \%$ ] and $17 \%$ [IQR $0.5 \%-50 \%$ ] in the rilpivirine and in the InSTI group, $p=0.087)$.

Overall, 62/466 (13.3\%) and 41/209 (19.6\%) patients in the rilpivirine and in the InSTI group discontinued at least one drug of the regimen for any reason. Discontinuations were due to toxicity in 34 (7\%) and in $17(8 \%)$ patients in the rilpivirine and in the InSTI group. Of the 51 discontinuations occurred because of toxicity, 28 (55\%) were deemed tenofovir toxicity (15 [3.2\%] and 13 [6.2\%] in the rilpivirine and in the InSTI group); other leading causes of discontinuation were non-tenofovir related toxicity or untoward drug interactions (19 [4.1\%] and 4 [1.9\%] in the rilpivirine and in the InSTI group) and treatment simplification (none and 12 [5.7\%] in the rilpivirine and in the InSTI group).

Non-tenofovir related toxicities leading to discontinuation were: liver toxicity in $9(2 \%)$, gastrointestinal toxicity in 5 (1\%), central nervous system toxicity in 4 (1\%) and undefined toxicity in one further patient in the rilpivirine group, liver toxicity in $1(<1 \%)$ and central nervous system toxicity in $1(<1 \%)$ in the InSTI group. Three $(1.5 \%)$ patients in the InSTI group discontinued for untoward drug interactions.

Of note, discontinuations occurred in 37/82 (45\%) patients who started raltegravir; 27 of these 37 patients 
Table 1 Baseline characteristics

\begin{tabular}{|c|c|c|c|c|}
\hline & Overall $(n=675)$ & InSTI + TDF/FTC $(n=209)$ & $\mathrm{RPV} / \mathrm{FTC} / \mathrm{TDF}(n=466)$ & $p$-value \\
\hline Age (years) & $46.2(39.9-51.6)$ & $48.7(41.8-53.3)$ & $45.4(39.2-50.7)$ & 0.0002 \\
\hline Male gender & $578(86 \%)$ & 174(83\%) & $404(87 \%)$ & 0.238 \\
\hline HIV risk factor & & & & $<0.0001$ \\
\hline MSM & $330(49 \%)$ & $78(37 \%)$ & $252(54 \%)$ & \\
\hline Heterosexual & 134(20\%) & $38(18 \%)$ & $96(21 \%)$ & \\
\hline IDU & $88(13 \%)$ & $49(23 \%)$ & $39(8 \%)$ & \\
\hline Other/Unknown & $123(18 \%)$ & $44(21 \%)$ & $79(17 \%)$ & \\
\hline Years since HIV diagnosis & $10.3(5.1-17.3)$ & $13.8(6.6-22.5)$ & $9.1(4.8-15.5)$ & $<0.0001$ \\
\hline History of AIDS defining events & $78(12 \%)$ & $26(12 \%)$ & $52(11 \%)$ & 0.696 \\
\hline Years of ART & $6.6(3.3-14.1)$ & $10.1(4.0-16.8)$ & $5.7(3.0-11.9)$ & $<0.0001$ \\
\hline NRTI-experience & $661(98 \%)$ & 206(99\%) & $455(98 \%)$ & 0.567 \\
\hline NNRTI-experience & $326(48 \%)$ & $88(42 \%)$ & $238(51 \%)$ & 0.037 \\
\hline Pl-experience & $533(79 \%)$ & 193 (92\%) & $340(73 \%)$ & $<0.0001$ \\
\hline Years with HIV-RNA <50copies/mL & $3.07(1.07-5.62)$ & $2.96(0.77-6.12)$ & $3.09(1.25-5.46)$ & 0.493 \\
\hline Time spent with residual viremia (\%) & $47.8(23.9-74.1)$ & $51.7(22.1-74.2)$ & $46.4(24.3-73.6)$ & 0.437 \\
\hline History of failure to NRTIs & $130(19 \%)$ & $70(34 \%)$ & $60(13 \%)$ & $<0.0001$ \\
\hline History of failure to NNRTIs & $32(5 \%)$ & $22(11 \%)$ & $10(2 \%)$ & $<0.0001$ \\
\hline History of failure to PIs & $110(16 \%)$ & $54(26 \%)$ & $56(12 \%)$ & $<0.0001$ \\
\hline Type of treatment & & & & $<0.0001$ \\
\hline PI-based & $438(65 \%)$ & $172(82 \%)$ & $266(57 \%)$ & \\
\hline NNRTI-based & $205(30 \%)$ & $24(12 \%)$ & 181 (39\%) & \\
\hline Other & $32(5 \%)$ & $13(6 \%)$ & $19(4 \%)$ & \\
\hline Nadir CD4+ count (cell/ $\mu \mathrm{L})$ & $271(160-384)$ & $228(122-336)$ & $289(205-397)$ & $<0.0001$ \\
\hline Zenith HIV-RNA before starting ART ( $\log _{10}$ copies $/ \mathrm{mL}$ ) & $4.85(4.08-5.32)$ & $4.97(4.11-5.40)$ & $4.80(4.08-5.29)$ & 0.187 \\
\hline 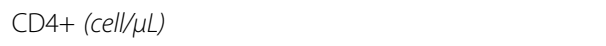 & $679(517-868)$ & $663(451-854)$ & $687(542-870)$ & 0.017 \\
\hline $\mathrm{HCV}-\mathrm{Ab}+$ & $159(24 \%)$ & $82(39 \%)$ & $77(17 \%)$ & $<0.0001$ \\
\hline ALP (U/L) & $87(69-106)$ & $93(73-110)$ & $86(69-105)$ & 0.040 \\
\hline $\operatorname{ALT}(U / L)$ & $31(22-47)$ & $34(22-63)$ & $30(22-43)$ & 0.001 \\
\hline Total bilirubin (mg/dL) & $0.57(0.34-1.44)$ & $0.68(0.41-1.71)$ & $0.50(0.32-1.37)$ & 0.003 \\
\hline FIB-4 & $0.89(0.65-1.27)$ & $1.08(0.72-1.64)$ & $0.84(0.61-1.12)$ & $<0.0001$ \\
\hline $\operatorname{eGFR}\left(\mathrm{ml} / \mathrm{min} / 1.73 \mathrm{~m}^{2}\right)$ & $104(93-113)$ & $102(90-111)$ & $105(95-114)$ & 0.013 \\
\hline Proteinuria (mg/dL) & $5(0-10)$ & $5(0-10)$ & $5(0-10)$ & 0.858 \\
\hline Total cholesterol (mg/dL) & $191(162-219)$ & $191(159-216)$ & $191(164-222)$ & 0.168 \\
\hline LDL cholesterol (mg/dL) & $119(95-144)$ & $117(91-145)$ & $120(97-144)$ & 0.407 \\
\hline HDL cholesterol (mg/dL) & $45(38-55)$ & $42(36-51)$ & $47(40-58)$ & $<0.0001$ \\
\hline Total/HDL cholesterol & $4.25(3.37-5.18)$ & $4.56(3.43-5.23)$ & $4.18(3.36-5.07)$ & 0.045 \\
\hline Triglycerides (mg/dL) & $122(86-180)$ & $137(98-215)$ & $117(82-166)$ & 0.0002 \\
\hline Glucose (mg/dL) & 85 (78-93) & $87(80-96)$ & $84(78-91)$ & 0.001 \\
\hline Hemoglobin $\left(10^{9} / L\right)$ & $15.1(14.1-15.7)$ & $14.9(14.1-15.7)$ & $15.1(14.2-15.7)$ & 0.315 \\
\hline Phosphate (mmol/L) & $0.98(0.86-1.10)$ & $0.99(0.85-1.13)$ & $0.98(0.87-10.8)$ & 0.621 \\
\hline
\end{tabular}

ALP Alkaline phosphatase, ALT Alanine aminotransferase, ART Antiretroviral therapy, eGFR Estimated glomerular filtration rate, FIB-4 Liver fibrosis-4 index, $H C V$ - $A b$ Anti-hepatitis C antibodies, HDL High density lipoprotein, IDU Intravenous drug user, InSTI Integrase strand transfer inhibitor, LDL Low density lipoprotein, MSM Man who have sex with men, NNRTI Non-nucleoside reverse transcriptase inhibitor, NRTIs Nucleoside reverse transcriptase inhibitors, PI Protease inhibitor, RPV/ FTC/TDF Rilpivirine/tenofovir disoproxil fumarate/emtricitabine, TDF/FTC Tenofovir disoproxil fumarate/emtricitabine 


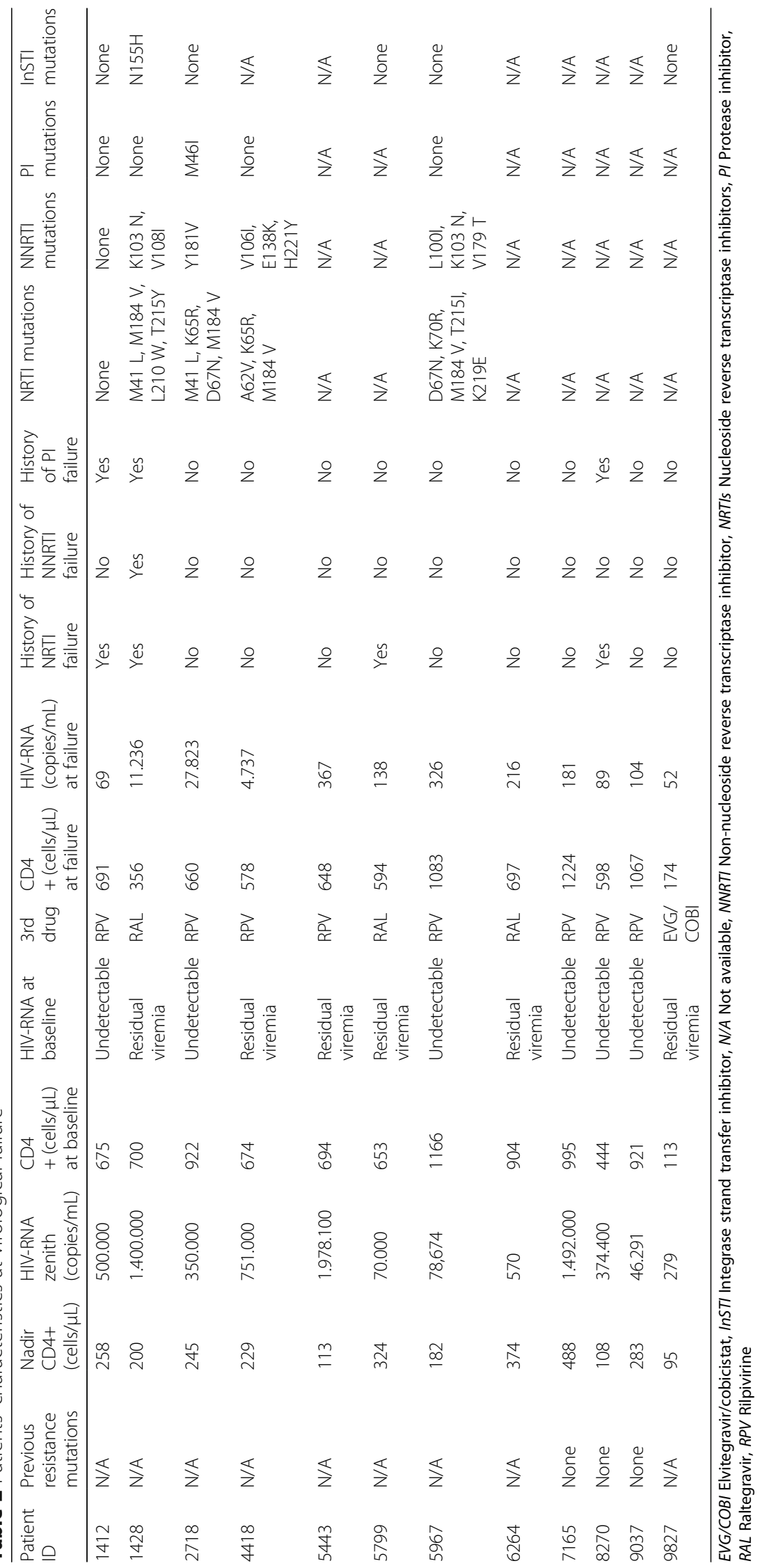




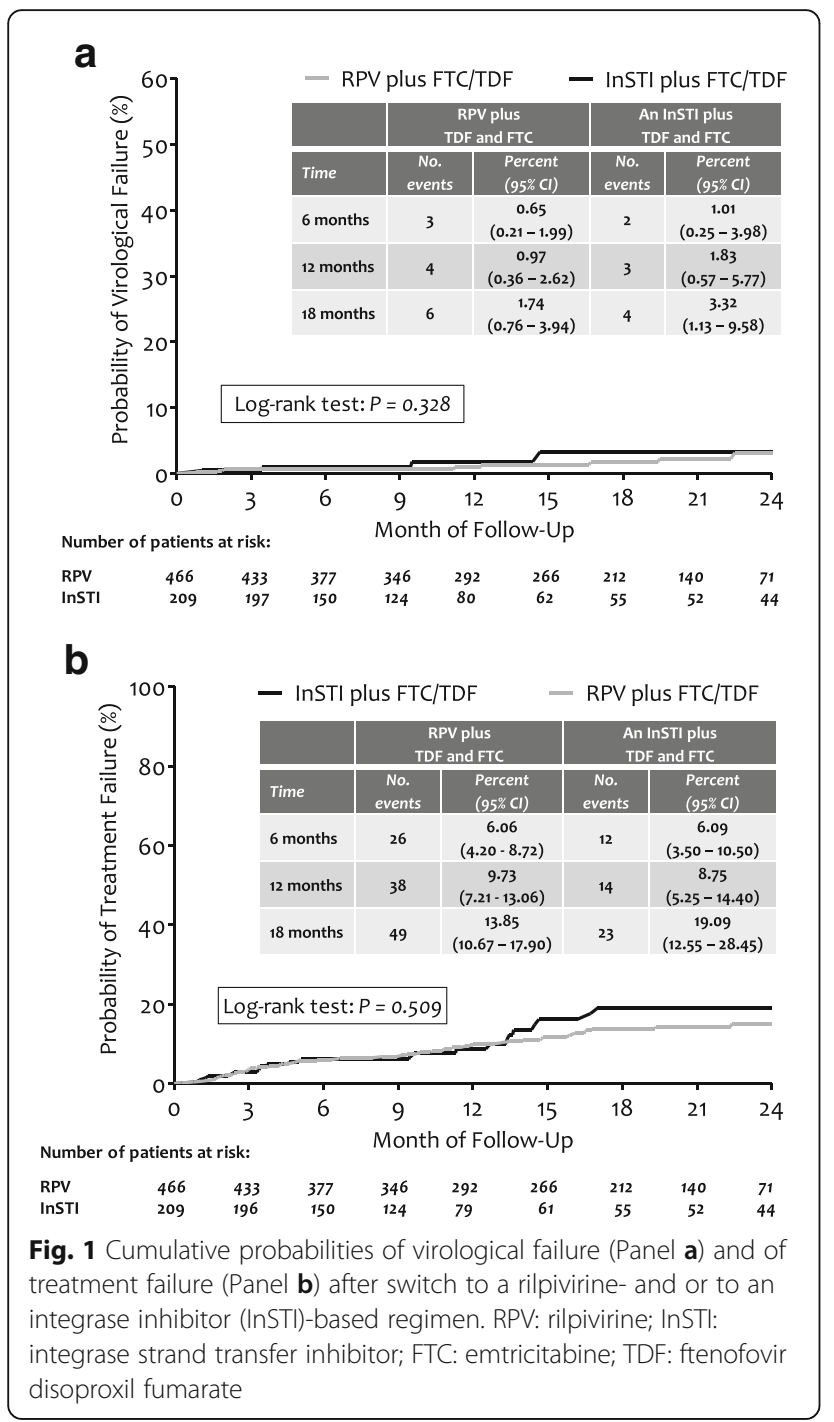

(73\%) discontinued only raltegravir; the main reason for raltegravir discontinuation was treatment simplification (12/27 [44\%]).

By the multivariable Cox regression model, TF was independently associated with being on therapy with a PI vs. a NNRTI at switch $(\mathrm{AHR}=0.52 ; 95 \% \mathrm{CI}=0.31-0.90$; $p=0.018)$, baseline total/HDL-cholesterol ratio $(\mathrm{AHR}=$ 1.19 per 0.5 -units increments; $95 \% \mathrm{CI}=1.06-1.34 ; p=$ 0.004 ), baseline eGFR (AHR $=0.78$ per 10 -units increments; $95 \% \mathrm{CI}=0.67-0.90 ; \quad p=0.001)$ and baseline hemoglobin $(\mathrm{AHR}=0.78$ per 1 -unit increments; $95 \% \mathrm{CI}$ $=0.64-0.94 ; p=0.009$ ), whereas treatment group (rilpivirine vs. InSTI), $\mathrm{HCV}$-coinfection, nadir and baseline CD4+ cell count, time with HIV-RNA $<50$ copies/mL, time spent with residual viremia, years of antiretroviral therapy, failure to NRTIs, failure to NNRTIs, failure to PIs, triglycerides and FIB-4 were not. No violation of the proportional hazard assumption was detected using graphical representation (Log-log plot).

\section{Discussion}

In this non-randomized study, the efficacy of switching to a FDC of rilpivirine/tenofovir disoproxil fumarate/emtricitabine was similar to that of switching to an InSTI plus tenofovir disoproxil fumarate/emtricitabine-based regimen: both the virological outcomes and treatment discontinuations were similar. As regards to virological outcomes, not only the cumulative risk of virological failure was very low and similar for both regimens, but also the incidence of viral blips and the exposure to residual viremia during follow-up were not statistically different. This suggest that both of these types of switch regimen can be safely used in clinical practice.

However, among patients switched to InSTI, most discontinuation occurred in those switched to raltegravir: this suggests clinician should preferentially switch patients virologically suppressed to a once-daily regimen.

A lower risk of failure was observed in patients switched from PIs: our hypothesis is that patients switched from a PI to a PI-sparing regimen have a greater improvement in symptoms than those switched from NNRTI to another NNRTI or to a InSTI-based regimen. Indeed, the only switch study in which the superiority of the switch strategy was demonstrated was the STRATEGY-PI [6]: in this randomized clinical trial, patients who underwent treatment switch to a FDC of elvitegravir/cobicistat/tenofovir disoproxil fumarate/ emtricitabine had a significant improvement in several PI-related symptoms (mainly gastrointestinal). On the contrary, in studies with comparable design, switching from a NNRTI to a FDC of elvitegravir/cobicistat/tenofovir disoproxil fumarate/emtricitabine was not superior to continuing the NNRTI [4], as well as switching from a FDC of efavirenz/tenofovir disoproxil fumarate/emtricitabine to rilpivirine/tenofovir alafenamide/emtricitabine did not resulted superior to continuing efavirenz/ tenofovir disoproxil fumarate/emtricitabine [2].

Although nonspecific, hemoglobin is a marker of the general health status; it has been also associated with mortality in HIV-infected patients [10]: thus, it not surprising that in our analysis it was independently associated with TF, as it is conceivable that patients with a worse general health status are more prone to interrupt or change drugs for toxicity issues. Patients with a higher baseline total/HDL-cholesterol ratio are those with greater metabolic problems: it is likely that, in these cases, treatment was then further modified, in the attempt of normalizing the dyslipidemia. The greater risk of TF in patients with lower eGFR value is consistent with the observation that a major cause of TF in our study was toxicity due to TDF.

Overall, the results of our study confirm, in a large, unselected population, the safety and the efficacy of 
switch regimens based on either rilpivirine or InSTI demonstrated in randomized clinical trials [1-6], thus providing useful data for clinical decision making.

With this study, we also aimed at identifying clinical reasons for opting for an InSTI- rather than a rilpivirine-based switch strategy in everyday clinical practice; this because there are no data from randomized clinical trials guiding clinicians in this decision and the results from switch trials do not clear indicate which is the best switching strategy. The results of our analysis showed that we preferentially opted for InSTI in patients with HCV co-infection, a longer ART duration, a shorter time of HIV suppression, an ongoing treatment with PIs. Possible drivers of these preferences are likely related to the common belief that NNRTIs entail greater liver toxicity (at least among persons co-infected with HCV) [11-13], to the results of the STRATEGY-PI study (which showed the superiority of this strategy compared to continuing on a PI) [6] and to the belief that InSTI are more potent than NNRTIs, thus favoring this strategy in patients a shorter time of viral suppression when a decision on whether or not switching has to be taken.

Major limitations of this study are the lack of randomization and the fact that the patients switched to rilpivirine/tenofovir disoproxil fumarate/emtricitabine were different from those switched to an InSTI in many baseline clinical features; however, after adjustment for these differences, the multivariable analysis confirmed that switching to a regimen rather than the other was not independently associated to TF.

\section{Conclusions}

In our clinical practice, the durability of switch regimens based on rilpivirine and on InSTI (along with TDF and FTC) was comparable and both showed a very low probability of VF.

\section{Additional file}

Additional file 1: Specific Pls and NNRTls ongoing at baseline. (DOCX $12 \mathrm{~kb}$ )

\footnotetext{
Abbreviations

AHR: Adjuster hazard ratio; ALP: Alkaline phosphatase; ALT: Alanine aminotransferase; ART: Antiretroviral therapy; AST: Aspartate aminotransferase; Cl: Confidence interval; CKD-EPI: Chronic Kidney Disease Epidemiology Collaboration Equation; eGFR: Estimated glomerular filtration rate; EVG/COBI: Elvitegravir/cobicistat; FDC: Fixed dose combination; FIB4: Liver fibrosis-4 index; HCV-Ab: Anti-hepatitis C antibodies; HDL: High density lipoprotein; IDD-HSR: Infectious Diseases Department San Raffaele Hospital; IDU: Intravenous drug user; InSTI: Integrase strand transfer inhibitor; IQR: Interquartile range; IR: Incidence rate; LDL: Low density lipoprotein; MSM: Man who have sex with men; N/A: Not available; NNRTI: Nonnucleoside reverse transcriptase inhibitor; NRTIs: Nucleoside reverse transcriptase inhibitors; OR: Odds ratio; PCR: Polymerase chain reaction; PI: Protease inhibitor; PI/r: Ritonavir-boosted protease inhibitor; RAL: Raltegravir; RPV: Rilpivirine; RPV/FTC/TDF: Rilpivirine/tenofovir disoproxil fumarate/emtricitabine; TDF/FTC: Tenofovir disoproxil fumarate/emtricitabine; TF: Treatment failure; VF: Virological failure
}

\section{Acknowledgements}

None to declare.

\section{Funding}

This study did not receive specific funding: data were collected as part of the routine clinical activity.

\section{Availability of data and materials}

The datasets analyzed during the current study are available from the corresponding author on reasonable request.

\section{Authors' contributions}

NG1 conceived the study, collected data and wrote the manuscript. AP and LG performed the statistical analyses and contributed to the writing of the manuscript. SN, NG2, MR, MM, AC1, VS, AL and AC2 collected data and contributed to the writing of the manuscript. All authors have read and approved the manuscript.

\section{Ethics approval and consent to participate}

This study was approved by the ethics committee of the San Raffaele Scientific Institute; the patients provided written informed consent for scientific analysis of their clinical and laboratory data.

\section{Consent for publication}

Not applicable.

\section{Competing interests}

Nicola Gianotti has been advisor for Gilead Sciences, AbbVie and Janssen-Cilag and has received speakers' honoraria from Gilead Sciences, ViiV, Bristol-Myers Squibb, Merck Sharp and Dohme, Roche, AbbVie, Boehringer Ingelheim, and Janssen-Cilag and is a member of the editorial board of BMC Infectious Diseases; Adriano Lazzarin has been advisor for and has received speakers' honoraria from Bristol-Myers Squibb, ViiV AbbVie, GILEAD, Janssen-Cilag, Mylan; Vincenzo Spagnuolo as received speakers' honoraria from Gilead Sciences, Merck Sharp and Dohme and ViiV. Andrea Poli, Laura Galli, Silvia Nozza, Marco Merli, Nadia Galizzi, Marco Ripa, Alessia Carbone and Antonella Castagna have no potential conflict of interest to declare.

\section{Publisher's Note}

Springer Nature remains neutral with regard to jurisdictional claims in published maps and institutional affiliations.

\section{Author details}

${ }^{1}$ Infectious Diseases, San Raffaele Scientific Institute, Via Stamira d'Ancona 20, 20127 Milan, Italy. ${ }^{2}$ Università Vita-Salute San Raffaele, Milan, Italy.

Received: 13 June 2017 Accepted: 12 November 2017

Published online: 16 November 2017

\section{References}

1. Palella FJ Jr, Fisher M, Tebas P, Gazzard B, Ruane P, Van Lunzen J, et al. Simplification to rilpivirine/emtricitabine/tenofovir disoproxil fumarate from ritonavir-boosted protease inhibitor antiretroviral therapy in a randomized trial of HIV-1 RNA-suppressed participants. AIDS. 2014;28:335-44.

2. DeJesus E, Ramgopal M, Crofoot G, Ruane P, LaMarca A, Mills A, Martorell $C T$, de Wet J, Stellbrink HJ, Molina JM, Post FA, Valero IP, Porter D, Liu Y, Cheng A, Quirk E, SenGupta D, Cao H. Switching from efavirenz, emtricitabine, and tenofovir disoproxil fumarate to tenofovir alafenamide coformulated with rilpivirine and emtricitabine in virally suppressed adults with HIV-1 infection: a randomised, double-blind, multicentre, phase 3b, non-inferiority study. Lancet HIV. 2017;4:e205-13.

3. Martínez E, Larrousse M, Llibre JM, Gutiérrez F, Saumoy M, Antela A, et al. Substitution of raltegravir for ritonavir-boosted protease inhibitors in HIVinfected patients: the SPIRAL study. AIDS. 2010;24:1697-707.

4. Pozniak A, Markowitz M, Mills A, Stellbrink HJ, Antela A, Domingo P, et al. Switching to coformulated elvitegravir, cobicistat, emtricitabine, and tenofovir versus continuation of non-nucleoside reverse transcriptase inhibitor with emtricitabine and tenofovir in virologically suppressed adults with HIV (STRATEGY-NNRTI): 48 week results of a randomised, open-label, phase 3b non-inferiority trial. Lancet Infect Dis. 2014;14:590-9. 
5. Trottier B, Lake JE, Logue K, Brinson C, Santiago L, Brennan C, Koteff JA, Wynne B, Hopking J, Granier C, Aboud M. Dolutegravir/abacavir/lamivudine versus current ART in virally suppressed patients (STRIIVING): a 48-week, randomized, non-inferiority, open-label, phase Illb study. Antivir Ther. 2017. doi:10.3851/IMP3166 [Epub ahead of print].

6. Arribas JR, Pialoux G, Gathe J, Di Perri G, Reynes J, Tebas P, et al. Simplification to coformulated elvitegravir, cobicistat, emtricitabine, and tenofovir versus continuation of ritonavir-boosted protease inhibitor with emtricitabine and tenofovir in adults with virologically suppressed HIV (STRATEGY-PI): 48 week results of a randomised, open-label, phase 3b, noninferiority trial. Lancet Infect Dis. 2014;14:581-9.

7. Eron JJ, Young B, Cooper DA, Youle M, Dejesus E, Andrade-Villanueva J, et al. Switch to a raltegravir-based regimen versus continuation of a lopinavir-ritonavir-based regimen in stable HIV-infected patients with suppressed viraemia (SWITCHMRK 1 and 2): two multicentre, double-blind, randomised controlled trials. Lancet. 2010;375:396-407.

8. Levey AS, Stevens LA, Schmid CH, Zhang YL, Castro AF 3rd, Feldman HI, et al. A new equation to estimate glomerular filtration rate. Ann Intern Med. 2009;150:604-12.

9. Sterling RK, Lissen E, Clumeck N, Sola R, Correa MC, Montaner J, et al. Development of a simple noninvasive index to predict significant fibrosis in patients with HIV/HCV coinfection. Hepatology. 2006;43:1317-25.

10. Tate JP, Justice AC, Hughes MD, Bonnet F, Reiss P, Mocroft A, et al. An internationally generalizable risk index for mortality after one year of antiretroviral therapy. AIDS. 2013;27:563-72.

11. Servoss JC, Kitch DW, Andersen JW, Reisler RB, Chung RT, Robbins GK. Predictors of antiretroviral-related hepatotoxicity in the adult AIDS clinical trial group (1989-1999). J Acquir Immune Defic Syndr. 2006:43:320-3.

12. Brück S, Witte S, Brust J, Schuster D, Mosthaf F, Procaccianti M, et al. Hepatotoxicity in patients prescribed efavirenz or nevirapine. Eur J Med Res. 2008;13:343-8.

13. Soriano V, Puoti M, Garcia-Gascó P, Rockstroh JK, Benhamou Y, Barreiro P, et al. Antiretroviral drugs and liver injury. AIDS. 2008;22:1-13.

\section{Submit your next manuscript to BioMed Central and we will help you at every step:}

- We accept pre-submission inquiries

- Our selector tool helps you to find the most relevant journal

- We provide round the clock customer support

- Convenient online submission

- Thorough peer review

- Inclusion in PubMed and all major indexing services

- Maximum visibility for your research

Submit your manuscript at www.biomedcentral.com/submit 